\title{
A Comparison of Direct Colorimetry of Titanium Pig- ments With Their Indirect Colorimetry Based on Spectrophotometry and a Standard Observer
}

\author{
By Deane B. Judd
}

\begin{abstract}
Recent work by Jacobsen in the colorimetry of titanium pigments has indicated that the ICI standard observer weights too lightly the spectral region below 430 millimicrons to accord with visual perception by average observers. This paper presents a repetition of this work for a single pair of titanium-pigment paints whose difference was measured by spectrophotometric means and then by seven observers by means of a visual colorimeter. Four of the seven observers corroborated Jacobsen's conclusion; three checked closely the standard observer. A modified standard observer based upon the spectral luminosity determinations of Gibson and Tyndall and Wald has been derived and is shown to account closely for the settings of the group of four observers that disagreed with the standard observer.
\end{abstract}

\section{Introduction}

It has recently been reported by Jacobsen $[1]^{1}$ that the ICI standard observer [2] apparently does not weight the far blue region of the spectrum in accordance with visual perception. This report is based upon an attempt to apply the spectrophotometer to the colorimetry of certain of the well-known near-white anatase and rutile titanium dioxide pigments when incorporated in a paint vehicle. The ICI $(x, y)$-chromaticity coordinates of these two paints were found to be essentially identical, although the spectrophotometric curves show marked differences in the spectral region below $430 \mathrm{~m} \mu$, and the paints exhibited visual color differences. It is suggested by Jacobsen that the ICI standard observer weights too lightly the spectral region below $430 \mathrm{~m} \mu$.

This suggestion agrees with two previous reports. It was pointed out by Judd [3] that an empirical formulation for chromaticity sensibility (the uniform-chromaticity-scale triangle) broke down badly for two observers unless the standard luminosity function be replaced for these observers (Priest, Brickwedde) by the Gibson-Tyndall experimental mean [4] between 400 and $440 \mathrm{~m} \mu$. This experimental mean is higher than the ICI

1 Figures in brackets indicate the literature references at the end of this paper. standard luminosity function by factors of 1.9 and 2.8 at 440 and $430 \mathrm{~m} \mu$, respectively, and the extrapolated values are increasingly higher toward $400 \mathrm{~m} \mu$ at which wavelength the ratio is more than 10. It was reported later by Wald [5] that the reciprocals of the absolute foveal thresholds, although agreeing fairly well in other parts of the spectrum, depart widely in the short-wave end of the spectrum from the ICI luminosity function. He states, "At $436 \mathrm{~m} \mu$ they reveal an average sensitivity of about 2.4 times, and at $405 \mathrm{~m} \mu$ about 9 times as high as the ICI factors indicate."*

Table 1 gives the relative spectral luminosities read from the curve published by Wald and compares them with the Gibson-Tyndall experimental mean and with the standard luminosity function.

It may be seen from this table that the indirect evidence by Judd and the direct determinations by Wald (both referring to fields of $2^{\circ}$ or less) combine to indicate that the Gibson-Tyndall experimental mean was given too little weight in adopting values for the standard luminosity function [6]. Thus these two reports are in qualitative agreement with Jacobsen's more recent suggestion.

*A recent paper by Weaver (J. Opt. Soc. Am. 39, 278; 1949) likewise reports spectral luminosities considerably higher, between 400 and $450 \mathrm{~m} \mu$, than the standard luminosity function. 
TABLE 1. Relative spectral luminosity

\begin{tabular}{|c|c|c|c|}
\hline $\begin{array}{l}\text { Wave- } \\
\text { length }\end{array}$ & ICI & Wald & $\begin{array}{l}\text { Gibson- } \\
\text { Tyndall }\end{array}$ \\
\hline$m_{\mu}$ & & & \\
\hline 370 & ...... & 0.00005 & -.... \\
\hline 380 & 0.0000 & .0004 & ...... \\
\hline 390 & .0001 & .0014 & - . \\
\hline 400 & .0004 & .004 & a 0.005 \\
\hline 410 & .0012 & .009 & a. 012 \\
\hline 420 & .0040 & .018 & a. 022 \\
\hline 430 & .0116 & .032 & .033 \\
\hline 440 & .023 & .045 & .043 \\
\hline 450 & .038 & .050 & .051 \\
\hline
\end{tabular}

a Extrapolated by Gibson and Tyndall.

It is the purpose of this paper to determine whether Jacobsen's conclusion is in quantitative, as well as qualitative, agreement with Wald's, and to report on the results of a repetition, by somewhat more refined methods, of Jacobsen's study of titanium-pigment paints.

\section{Colorimetric and Spectrophotometric Study of Two Samples of Titanium- Pigment Paints}

\section{Preparation of Specimens}

The samples of rutile and anatase forms of titanium dioxide pigment ${ }^{2}$ were rubbed up with linseed oil in accord with standard procedures. ${ }^{3}$ The resulting pastes were used to fill a cylindrical cavity, $1 \mathrm{~mm}$ deep, in aluminum holders, and were placed in optical contact with clear cover glasses cut from nearby parts of the same sheet.

\section{Spectrophotometric Measurements}

The spectral reflectances of these two specimens relative to freshly prepared magnesium oxide were measured between 400 and $750 \mathrm{~m} \mu$ on an automatic recording spectrophotometer, ${ }^{4}$ the specularly reflected energy from the cover glass being eliminated from measurement by insertion of a black velvet plug in the porthole of the sphere corresponding to the direction of mirror reflection. Corrections for zero, 100-percent, and wavelengthscale errors were applied [7]. Spectral directional $\left(45^{\circ} 0^{\circ}\right)$ reflectances relative to magnesium oxide

\footnotetext{
2 Supplied from the Research Laboratory, Titanium Division, National Lead Company, by Jacobsen.

${ }^{3}$ Under the direction of E. F. Hickson of this Bureau.

4 Measurements made by H. J. Keegan of this Bureau.
}

were also measured for similar specimens prepared from the same pastes on the Beckman spectrophotometer [8] over the wavelength range 350 to $400 \mathrm{~m} \mu .^{5} \quad$ These reflectances were multiplied by a constant (nearly equal to one) so that the product agreed with the spectral reflectances found on the recording spectrophotometer at $400 \mathrm{~m} \mu$, and the corresponding products were adopted for the spectral range 350 to $400 \mathrm{~m} \mu$. Table 2 and figure 1 give the results of these measurements.

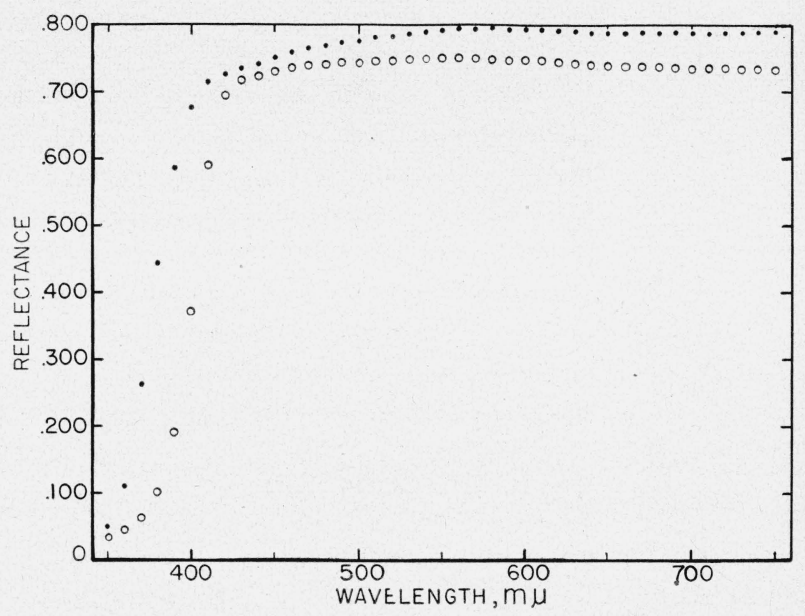

FIGURE 1. Spectral reflectance of pastes made by rubbing up in linseed oil two forms of titanium dioxide pigment.

- Anatase+cover glass; $\bigcirc$, rutile+cover glass.

Both pastes were in contact with idential cover glasses during measurement. Note that the rutile form yields much lower spectral reflectance in the neighborhood of $400 \mathrm{~m} \mu$.

Figure 1 shows that our measurements duplicate the essential features of the spectrophotometric curves published by Jacobsen, but differ in two respects; first, the curve for rutile is everywhere lower than that for anatase, particularly in the long-wave end; second, both curves decline slightly toward the long-wave end. The first difference suggests that the standard procedure of rubbing up the pigment in linseed oil with a glass muller has darkened the rutile paste more than the procedures used by Jacobsen. The second difference is ascribable to the cover glasses. Because of multiple reflections between the pigment-oil paste and the under side of the front face of the cover glass, any slight absorption of energy by the cover glass increasing with wavelength may produce an appreciable effect.

5 Measurements made by Marion A. Belknap of this Bureau. 
TABLE 2. Spectral directional reflectances of rutile and anatase titanium dioxide pigments in oil behind a cover glass.

\begin{tabular}{|c|c|c|}
\hline \multirow{2}{*}{ Wavelength } & \multicolumn{2}{|c|}{$\begin{array}{l}\text { Spectral directional } \\
\text { reflectance }\end{array}$} \\
\hline & Rutile & Anatase \\
\hline$m \mu$ & & \\
\hline $350^{\circ}$ & 0.033 & 0.049 \\
\hline 330 & .045 & .111 \\
\hline 370 & .063 & .262 \\
\hline 380 & .101 & .444 \\
\hline 390 & .191 & .586 \\
\hline 400 & .371 & .676 \\
\hline 410 & .591 & .714 \\
\hline 420 & .694 & .726 \\
\hline 430 & .717 & .735 \\
\hline 440 & .724 & .742 \\
\hline 450 & .730 & .751 \\
\hline 460 & .736 & .758 \\
\hline 470 & .739 & .765 \\
\hline 480 & .741 & .769 \\
\hline 490 & .743 & .773 \\
\hline 500 & .744 & .776 \\
\hline 510 & .745 & .780 \\
\hline 520 & .746 & .783 \\
\hline 530 & .748 & .786 \\
\hline 540 & .749 & .789 \\
\hline 550 & .750 & .792 \\
\hline 560 & .751 & .794 \\
\hline 570 & .750 & .794 \\
\hline 580 & .749 & .794 \\
\hline 590 & .747 & .793 \\
\hline 600 & .746 & .792 \\
\hline 610 & .746 & .792 \\
\hline 620 & .744 & .791 \\
\hline 630 & .742 & .790 \\
\hline 640 & .740 & .788 \\
\hline 650 & .739 & .788 \\
\hline 660 & .738 & .788 \\
\hline 670 & .738 & .788 \\
\hline 680 & .737 & .788 \\
\hline 690 & .737 & .789 \\
\hline 700 & .735 & .788 \\
\hline 710 & .736 & .788 \\
\hline 720 & .735 & .788 \\
\hline 730 & .734 & .788 \\
\hline 740 & .734 & .788 \\
\hline 750 & .734 & .789 \\
\hline
\end{tabular}

\section{Colorimetric Measurements}

The same specimens (pigment-oil behind cover glass) that were measured on the recording spectrophotometer were compared on a chromaticitydifference colorimeter [9] on the same and following day by seven observers. The luminator used consists of an incandescent-filament projection lamp combined with Corning daylite glass of such thickness as to produce closely a correlated color temperature of $6,800^{\circ} \mathrm{K}$. The relative energy distribution of this source is given in the Report of the OSA Committee on Colorimetry [10]. The observing field has a double trapezoid pattern and subtends $13^{\circ}$ vertically and $9^{\circ}$ horizontally at the eye of the observer. The difference in the settings of the wedges of the colorimeter, corresponding for each observer to the chromaticity differences between the rutile and anatase specimen, were converted to differences in the chromaticity coordinates $(x, y)$ of the ICI system by calibration graphs based on the luminator used (Macbeth $\left.6,800^{\circ} \mathrm{K}\right)$. These $(x, y)$-differences (rutile minus anatase) have been applied to the $(x, y)$-chromaticity coordinates of the anatase computed in the usual way from the ICI standard observer, thus yielding experimental determinations for the chromaticity coordinates $(x, y)$ of the rutile specimen. Figure 2 compares these results

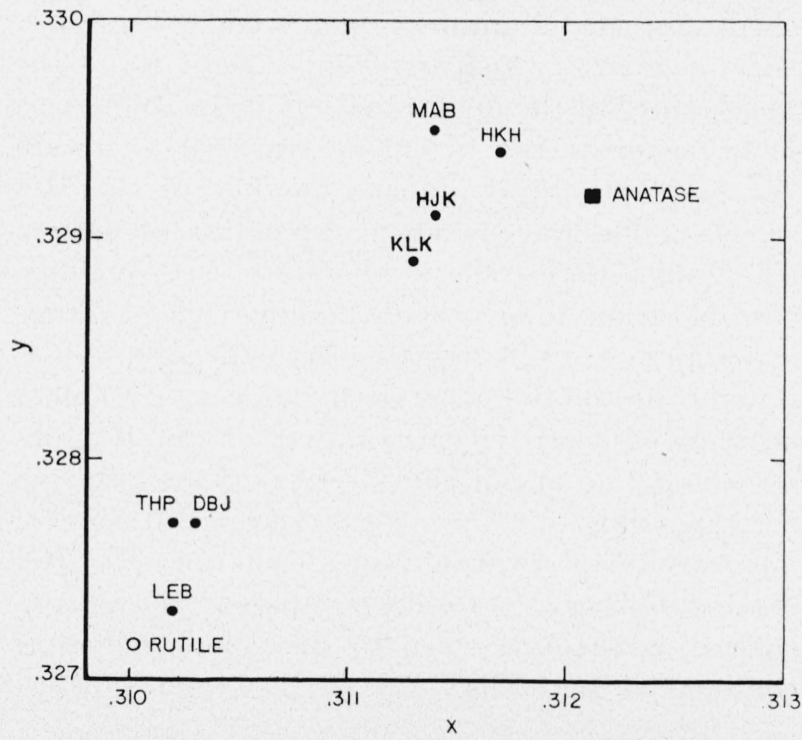

Figure 2. Chromaticity of specimens of titanium pigment in oil under cover glass.

Observer: ICI Staydard; luminator; Macbeth $6,800^{\circ} \mathrm{K}$; $\bigcirc$, compute 1 from spectral reflectance via $\mathrm{IC} i$ observer; observe 1 relative to anatase.

Note that three of the observers (THP, DBJ, LEB) find the chromaticity difference between the anatase and rutile forms to be closely in accord with that computed by means of the ICI standard observer; but the other four observers (MAB, HKH, HJK, KLK) do not.

with the chromaticity coordinates found for the rutile specimen from its spectral reflectance by the usual computation based on the ICI standard observer. On this extended scale the chromaticity of the luminator $(x=0.3082, y=0.3228$ ) would be 
represented rather far off the graph, the specimens being yellowish whites.

It will be noted immediately from figure 2 that there is a considerable individual observer difference in this determination. The chief differences lie along the straight line passing through the short-wave portion of the spectrum. This difference is to be expected among observers having varying, amounts of (yellowish) ocular pigmentation. It may be noted also that the observers divide into two groups. This division into two groups was unexpected, and may be statistically nonsignificant because of the small number of observers. One group of three observers, however, yields a fairly good check of the result computed by the ICI standard observer, the maximum discrepancy being a $y$-difference of 0.0005 , only slightly higher than the estimated uncertainty (0.0003) of the determination of the difference on the chromaticity-difference colorimeter. The other group of four observers yields a very poor check, the maximum discrepancy being a $y$-difference of 0.0023 , which is seven or eight times the uncertainty of the determination of the difference. It is assumed that the observers used to obtain Jacobsen's result of nonadaptability of the ICI standard observer would correspond to this group. The result of this check, therefore, is a qualified corroboration of Jacobsen's finding. Our average experimental result deviates from the result computed from the ICI observer by indicating a higher short-wave sensitivity than that of the ICI observer, and no one of our observers made a setting corresponding to a lower sensitivity. But some of our observers checked the result of the ICI standard observer to a degree that would be considered satisfactory even to the color graders of commercial materials.

\section{Modification of the ICI Standard Observer}

There remains yet to discover whether the experimental result of Wald based on 22 observers of the average age of 20 , which agrees qualitatively with Jacobsen's finding, also agrees quantitatively. The qualitative check suggests that the standard luminosity function is nonrepresentative in the sense that it corresponds to an observer having too heavy an ocular pigmentation (yellowish) to correspond to the average. It is proposed, therefore, to derive the tristimulus values of the spectrum for an observer differing from the ICI standard by having such ocular pigmentation as would change his luminosity function from the standard so as to agree with the Wald-GibsonTyndall experimental average (see table 1). These distribution functions are then to be applied to the spectral reflectances of table 2 , in place of the distribution functions of the standard observer, to see whether they will result in a check of the second group of observers in the same way as the standard observer has checked the first group.

If $\bar{y}^{\prime}$ be the modified luminosity function, this check could be obtained by computing modified $\bar{x}$ - and $\bar{z}$-functions defined as:

$$
\bar{x}^{\prime}=\bar{y}^{\prime}(x / y) \text {, and } \bar{z}^{\prime}=\bar{y}^{\prime}(z / y),
$$

where $x \equiv \bar{x} /(\bar{x}+\bar{y}+\bar{z}), \quad y \equiv \bar{y} /(\bar{x}+\bar{y}+\bar{z}), \quad$ and $z \equiv$ $\bar{z} /(\bar{x}+\bar{y}+\bar{z})$.

The chromaticity diagram generated by this $\left(\bar{x}^{\prime}, \bar{y}^{\prime}, \bar{z}^{\prime}\right)$-system, however, would differ grossly from the $(x, y)$-chromaticity diagram, the equienergy point, for example, being displaced far from the center of the Maxwell triangle. This gross difference would make difficult the interpretation of any computed results. It has seemed worthwhile, therefore, to define the modified distribution functions $\left(\bar{x}_{g}, \bar{y}_{g}, \bar{z}_{g}\right)$ by a further linear, homogeneous transformation so as to make the functions and the chromaticity diagram as similar as possible to the ICI functions and diagram. Define, then:

$$
\left.\begin{array}{c}
\bar{x}_{g}=k_{1} \bar{x}^{\prime}+k_{2} \bar{y}^{\prime}+k_{3} \bar{z}^{\prime} \\
\bar{y}_{g}=k_{4} \bar{x}^{\prime}+k_{5} \bar{y}^{\prime}+k_{6} \bar{z}^{\prime} \\
\bar{z}_{g}=k_{7} \bar{x}^{\prime}+k_{8} \bar{y}^{\prime}+k_{9} \bar{z}^{\prime}
\end{array}\right\}
$$

where $k_{1}$ to $k_{9}$ are constants independent of wavelength satisfying the following conditions:

(a) $\bar{y}_{g}$ shall be as closely like $\bar{y}$ as possible. There is no reason why $\bar{y}_{g}$ should depart from $\bar{y}$ at all except in the spectral range $460 \mathrm{~m} \mu$ and below. Set, therefore, $k_{4}=k_{6}=0$ and $k_{5}=1$. This makes $\bar{y}_{g}=\bar{y}^{\prime}$.

(b) $\bar{z}_{g}$ shall be as closely like $\bar{z}$ as possible. This condition is met by setting $k_{7}=k_{8}=0$, making $\bar{z}_{g}=k_{9} \bar{z}^{\prime}$. 
(c) $\bar{x}_{g}$ shall be as closely like $\bar{x}$ as possible. This condition is closely met by setting the chromaticity coordinate $x_{g}=\bar{x}_{g} /\left(\bar{x}_{g}+\bar{y}_{g}+\bar{z}_{g}\right)=$ 0.0070 at $500 \mathrm{~m} \mu$, that is, by making the modified $\bar{x}$-function be nearly zero at $500 \mathrm{~m} \mu$ like the original $\bar{x}$-function, but still large enough to prevent the modified $x$-function from being anywhere less than zero.

(d) The equienergy point on the modified system shall be at $x_{g}=y_{g}=z_{g}=1 / 3$, like that of the modified system.

(e) The $\bar{x}_{g}$ and $\bar{y}_{g}$ functions shall have the same long-wave crossing point (about $578.1 \mathrm{~m} \mu$ ) as the $\bar{x}$ and $\bar{y}$ functions.

The constants, $k_{1}$ to $k_{9}$, meeting these requirements have been found by solution of simultaneous equations, and we may write the definitions of $\bar{x}_{g}, \bar{y}_{g}$ from eq1 and 2 as follows:

$$
\left.\begin{array}{l}
\bar{x}_{g} \equiv 0.9310 \bar{y}^{\prime}(x / y)+0.0688 \bar{y}^{\prime}-0.0866 \bar{y}^{\prime}(z / y) \\
\bar{y}_{g} \equiv+1.0000 \bar{y}^{\prime} \\
\bar{z}_{g} \equiv+0.5273 \bar{y}^{\prime}(z / y)
\end{array}\right\} \text {, }
$$

which is in terms simply of the revised luminosity function, $\bar{y}^{\prime}$, and the chromaticity coordinates $(x, y)$ of the ICI system.

Table 3 gives the tristimulus values of the spectrum $\left(\bar{x}_{g}, \bar{y}_{g}, \bar{z}_{g}\right)$ computed from eq 3 and also the chromaticity coordinates $\left(x_{g}, y_{g}\right)$. It shows, of course, the revised values of the luminosity function $\left(\bar{y}_{g}\right)$ on which the other two distribution functions are based. Comparison with table 1 will show that the revised luminosity function agrees well both with the Gibson-Tyndall and with the Wald experimental mean for the spectral region of $450 \mathrm{~m} \mu$ and below. For $470 \mathrm{~m} \mu$ and above it is identical with the standard luminosity function. Figure 3 compares the modified distribution functions with the standard functions, and figure 4 compares the spectrum locus of the modified chromaticity diagram with that of the standard $(x, y)$-diagram. The equienergy points of the two diagrams are, of course, coincidental, and it may be seen that, as intended, $x_{g}=0.007$ for 500 $\mathrm{m} \mu$, and the two loci also coincide at the long-wave crossing point of $x$ and $y$. Elsewhere there is considerable change in the spacing of the spectrum chromaticities, and Macbeth $6,800^{\circ} \mathrm{K}$ has been shifted from $x=0.3082, y=0.3228$ to $x_{g}=0.3113$, $y_{g}=0.3276$. These changes are caused by the temporary adoption of the revised luminosity function. Figure 3 shows that the comparatively minor change in this function (difference between $\bar{y}_{g}$ and $\bar{y}$ ) has generated a considerably larger change in the $x$-function and a rather drastic change in the $z$-function, making it, of course, considerably higher in the spectral region $430 \mathrm{~m} \mu$ and below as suggested by Jacobsen.

\begin{tabular}{|c|c|c|c|c|c|c|}
\hline \multirow{2}{*}{$\begin{array}{l}\text { Wave- } \\
\text { length }\end{array}$} & \multicolumn{3}{|c|}{ Tristimulus values } & \multicolumn{3}{|c|}{ Chromaticity coordinates } \\
\hline & $\bar{x}_{0}$ & $\bar{y}_{0}$ & $\bar{z}_{0}$ & $x_{0}$ & $y_{0}$ & $z_{0}$ \\
\hline$m \mu$ & & & & & & \\
\hline 360 & 0.0001 & 0.000005 & 0.0004 & $\ldots$ & $-\cdots$ & $\ldots$ \\
\hline 370 & .0009 & .00005 & .0042 & -... & --- & $\ldots$ \\
\hline 380 & .0072 & .0004 & .0346 & -..- & $\ldots$ & -... \\
\hline 390 & .0261 & .0014 & .1237 & $\ldots-$ & -... & $\ldots$ \\
\hline 400 & .0754 & .0040 & .3611 & 0.171 & 0.009 & 0.820 \\
\hline 410 & .1870 & .0100 & .9036 & .170 & .009 & .821 \\
\hline 420 & .3474 & .0200 & 1. 7028 & .168 & .010 & .822 \\
\hline 430 & .4003 & .0320 & 2. 0154 & .164 & .013 & .823 \\
\hline 440 & .3250 & .0430 & 1. 7154 & .156 & .021 & .823 \\
\hline 450 & .2175 & .0510 & 1. 2545 & .143 & .034 & .824 \\
\hline 460 & .1478 & .0680 & 0.9975 & .122 & .056 & .822 \\
\hline 470 & .0766 & .0910 & .6789 & .090 & .108 & .802 \\
\hline 480 & .0282 & .1390 & .4287 & .047 & .233 & .719 \\
\hline 490 & .0038 & .2080 & .2453 & .008 & .455 & .537 \\
\hline 500 & .0032 & .3230 & .1434 & .007 & .688 & .305 \\
\hline 510 & .0296 & .5030 & .0834 & .048 & .817 & .135 \\
\hline 520 & .1009 & .7100 & .0412 & .118 & .833 & .048 \\
\hline 530 & .2097 & .8620 & .0223 & .192 & .788 & .020 \\
\hline 540 & .3342 & .9540 & .0107 & .257 & .734 & .008 \\
\hline 550 & .4711 & .9950 & .0046 & .320 & .676 & .003 \\
\hline 560 & .6216 & .9950 & .0021 & .384 & .615 & .001 \\
\hline 570 & .7748 & .9520 & .0011 & .448 & .551 & .001 \\
\hline 580 & .9128 & .8700 & .0009 & .512 & .488 & .000 \\
\hline 590 & 1.0075 & .7570 & .0006 & .571 & .429 & .000 \\
\hline 600 & 1. 0322 & .6310 & $\ldots$ & .621 & .379 & .000 \\
\hline 610 & 0.9681 & .5030 & -.... & .658 & .342 & .000 \\
\hline 620 & .8217 & .3810 & ..... & .683 & .317 & .000 \\
\hline 630 & .6163 & .2650 & -.... & .699 & .301 & .000 \\
\hline 640 & .4290 & .1750 & ..... & .710 & .290 & .000 \\
\hline 650 & .2713 & .1070 & ..... & .717 & .283 & .000 \\
\hline 660 & .1577 & .0610 & ..... & -.. & ... & $\ldots$ \\
\hline 670 & .0836 & .0320 & $\ldots$. & .... & -... & -... \\
\hline 680 & .0448 & .0170 & ..... & .... & $\ldots$ & $\cdots$ \\
\hline 690 & .0217 & .0082 & -..... & .... & -... & -... \\
\hline 700 & .0109 & .0041 & -.... & ... & -... & -... \\
\hline 710 & .0055 & .0021 & -... & -.. & $\ldots$ & $\ldots$ \\
\hline 720 & .0028 & .00105 & ..... & .... & -... &.-- \\
\hline 730 & .0013 & .00052 & -... & .... & $\cdots$ & $\ldots$ \\
\hline 740 & .0007 & .00025 & $\ldots$ & -... & -..- & -..- \\
\hline 750 & .0003 & .00012 & - . & .... & -... & ... \\
\hline 760 & .0002 & .00006 & $\ldots$ & .... & - & .... \\
\hline 770 & .0001 & .00003 & $\ldots$ & .... & -... & $\ldots$ \\
\hline
\end{tabular}

TABLE 3. Tristimulus values and chromaticity coordinates of the spectrum in the modified ICI system 


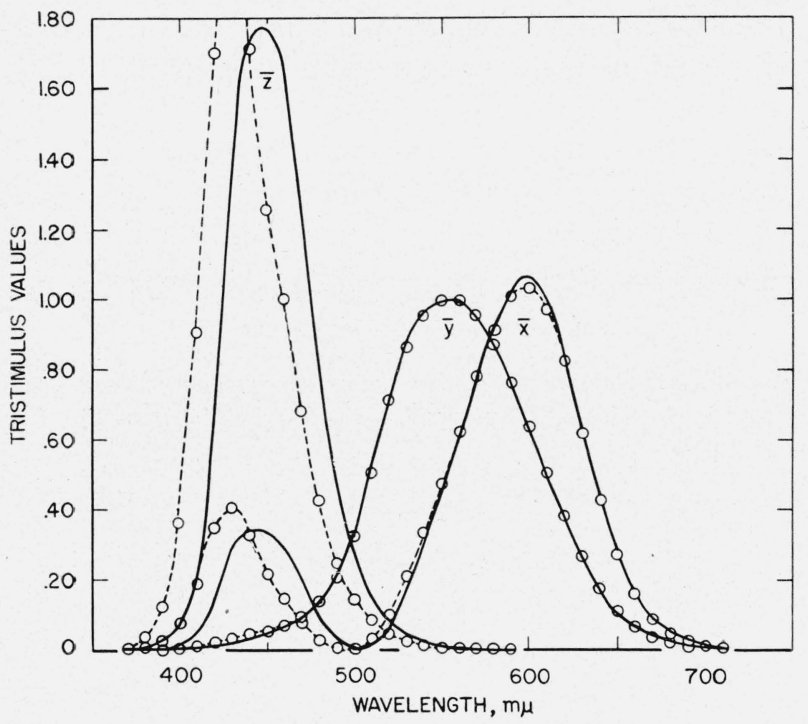

Figure 3. Comparison of the tristimulus values of the spectrum according to the ICI standard observer (solid lines) with those (dotted lines) according to an observer modified to agree with the Wald-Gibson-Tyndall data on spectral luminosity below $470 \mathrm{m \mu}$.

Note that the small changes introduced into the spectral luminosity $(\bar{y})$ function near the short-wave extreme require changes to be made in the other $(\bar{x}$ and $\bar{z})$ functions that are relatively large; for example, the peak of the $\bar{z}$. function is shifted by about $15 \mathrm{~m} \mu$ in the short-wave direction.

\section{Check of Modified Distribution Func- tions Against Experimental Results on Anatase and Rutile}

The first check of the modified distribution functions is to apply them in the same way as the standard distribution functions were applied to obtain the comparison given in figure 2 . The wedges of the chromaticity-difference colorimeter were recalibrated by means of the modified functions, the experimental results recomputed on that basis, and the chromaticity coordinates of the specimens (pigment in oil plus cover glass) recomputed in the $\left(\bar{x}_{g}, \bar{y}_{g}, \bar{z}_{g}\right)$ system. Figure 5 compares the computed chromaticity of the rutile specimen with experimental determinations of chromaticity of the rutile specimen found relative to the anatase specimen as standard. Table 4 gives the chromaticity coordinates from which figures 2 and 5 have been plotted. It will be seen that the modified distribution functions do indeed check the results by the second group of observers (KLK, HJK, $\mathrm{MAB}$ and $\mathrm{HKH}$ ) much as the standard ICI

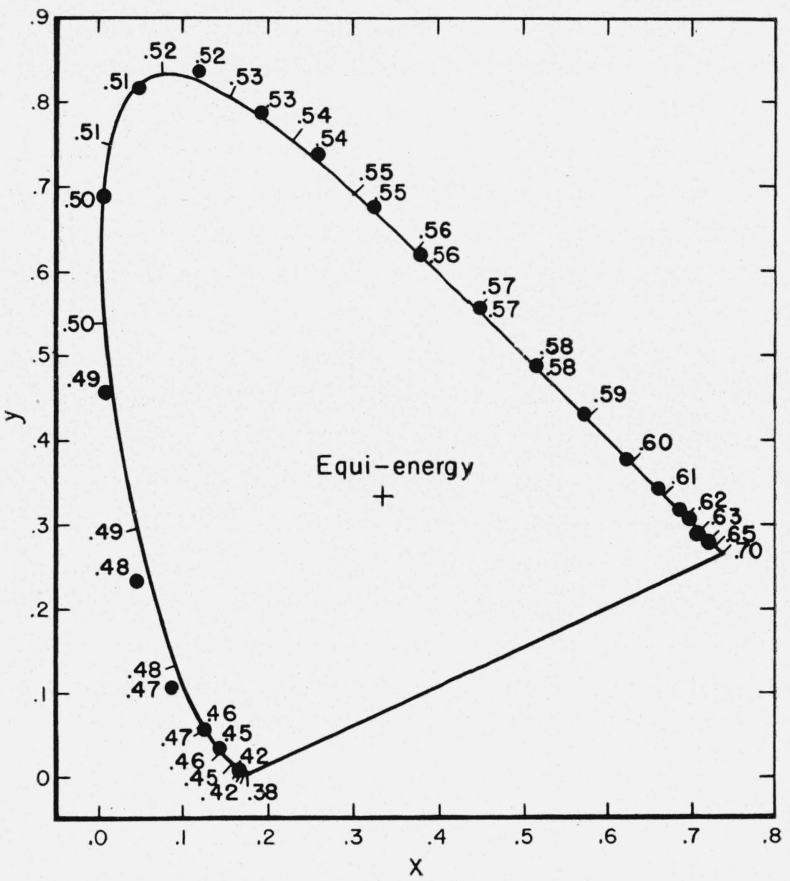

Figure 4. Comparison of the spectrum locus on the $(x, y)$ chromaticity plot of the ICI system with that (dots) on the corresponding plot of the modified ICI system.

The equienergy foint is set at $x=y=1 / 3$ in both systems. Note that the chromaticity region near the short-wave extreme of the spectrum has been expanded relative to other regions in the modified system.

observer checks the results by the first group of observers (LEB, DBJ, THP).

The second check of the modified distribution functions against the experimental results on anatase and rutile was carried out in such a way as to avoid even the presumably negligible uncertainties of applying the calibration of the wedges of the chromaticity-difference colorimeter. It is based simply on the average experimental result. This result may be summarized by saying that, if the rutile specimen be viewed through thicknesses of the yellow and green wedges corresponding to 90 and 36 wedge units, respectively, then the average of the seven observers will find that it has the same chromaticity as the anatase specimen viewed through thicknesses corresponding to 82 yellow- and 43 green-wedge units. The spectral transmittances of the pairs of wedges of these thicknesses have been computed from the known spectral transmittances of the yellow wedge at a thickness corresponding to 164 units and those of the green wedge at a thickness corresponding to 98 units [9]. These spectral transmittances have been multiplied by the spectral reflectances of the 


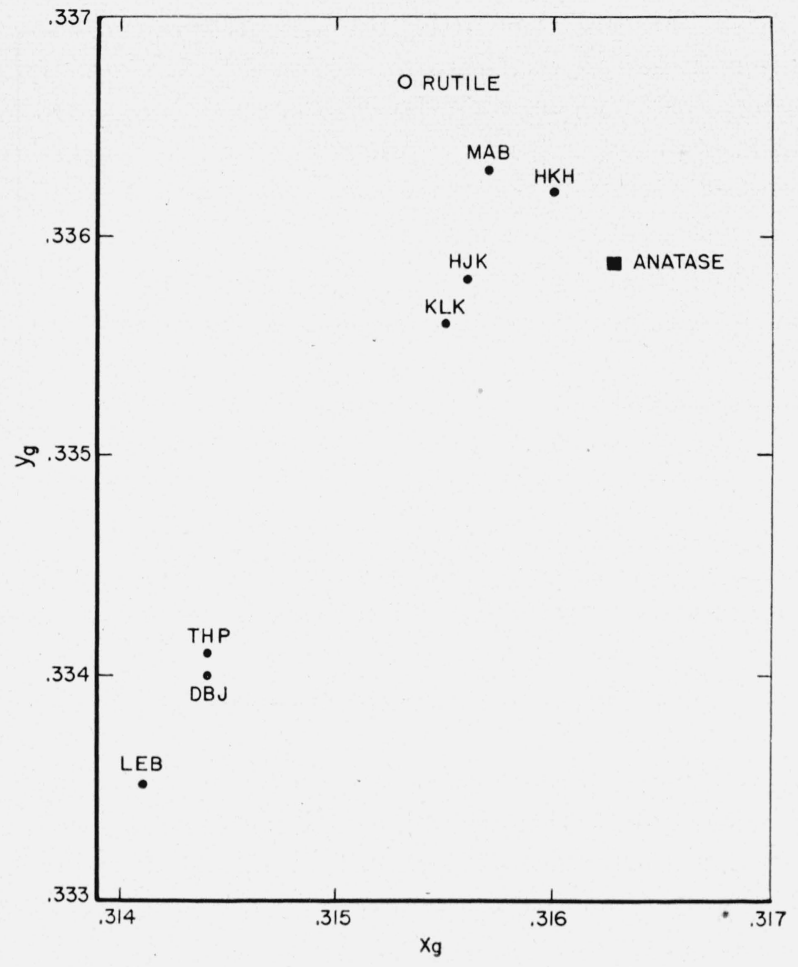

Figure 5. Chromaticity of specimens of titanium pigments in oil under cover glass.

Observer: modified ICI standard; luminator: Macbeth $6,800^{\circ} \mathrm{K}$; computed from spectral reflectance viả modified ICI observer relative to anatase.

Note that the spectrophotometric result by the modified observer agrees well with visual observations by the second group of observers (MA B, HKH, HJK, KLK) much in the same way as the ICI observer agreed with the first group (THP, DBJ, LEB).

titanium pigment specimens (see table 2) and by the relative spectral distribution of the luminator (Macbeth, $6,800^{\circ} \mathrm{K}$ ) that was used. These triple products give the spectral distributions of radiant flux that the seven observers on the average found to have identical chromaticities. These two spectral distributions of radiant flux, if adjusted by a constant factor to correspond to the same luminance, form for the average of these seven observers, therefore, what is known as a metameric pair. They are shown in figure 6 together with the spectral distribution of the luminator used (Macbeth $6,800^{\circ} \mathrm{K}$ ).

If a standard observer truly represented the average of the seven observers used in this study, use of that standard observer to compute chromaticity coordinates for these two spectral distributions should yield identical results. Both the standard observer and the modified standard observer have been subjected to this test. Figure

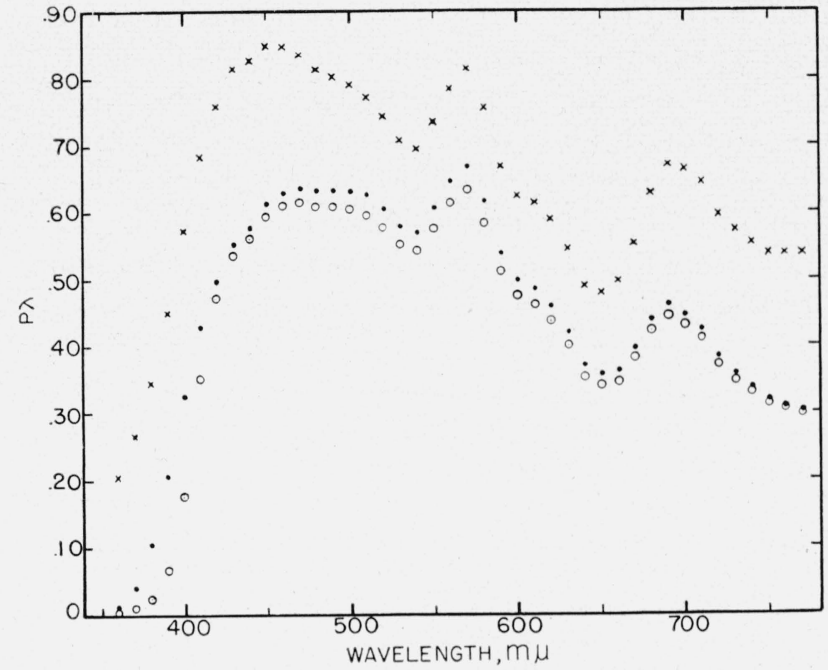

Figure 6. Spectral distributions of radiant flux.

$\mathrm{x}$, Macbeth daylight, color temperature $6,800^{\circ} \mathrm{K}$ used as source; , reflected from anatase plus cover glass, transmitted by thicknesses of yellowish and greenish glass, $90 \mathrm{Y}$ plus $36 \mathrm{G}$; $\bigcirc$, reflected from rutile plus cover glass, transmitted by thicknesses of yellowish and greenish glass, $82 \mathrm{Y}$ plus $43 \mathrm{G}$. The latter two distributions correspond to the same chromaticity according to the average of our seven observers.

TABLE 4. Chromaticity coordinates for titanium pigments in oil behind a cover glass

\begin{tabular}{|c|c|c|c|c|c|}
\hline \multirow{2}{*}{$\begin{array}{l}\text { Method of } \\
\text { evaluation }\end{array}$} & \multirow{2}{*}{$\begin{array}{l}\text { Form of } \\
\text { titanium } \\
\text { pigment }\end{array}$} & \multicolumn{2}{|c|}{$\begin{array}{l}\text { Chromaticity } \\
\text { difference }\end{array}$} & \multicolumn{2}{|c|}{$\begin{array}{c}\text { Chromaticity } \\
\text { coordinates }\end{array}$} \\
\hline & & $\Delta x$ & $\Delta y$ & $x$ & $y$ \\
\hline $\begin{array}{l}\text { Spectrophotomet- } \\
\text { ric. }\end{array}$ & $\left\{\begin{array}{l}\text { Rutile..... } \\
\text { Anatase... }\end{array}\right.$ & 0.0021 & 0.0020 & $\begin{array}{r}0.3100 \\
.3121\end{array}$ & $\begin{array}{r}0.3272 \\
.3292\end{array}$ \\
\hline $\begin{array}{l}\text { Colorimetric: } \\
\text { LEB } \\
\text { DBJ } \\
\text { THP }\end{array}$ & $\begin{array}{l}\text { Rutile } \ldots . . . . \\
. . . \text { do } \ldots . . . .\end{array}$ & $\begin{array}{l}.0019 \\
.0018 \\
.0019\end{array}$ & $\begin{array}{l}.0019 \\
.0015 \\
.0015\end{array}$ & $\begin{array}{l}.3102 \\
.3103 \\
.3102\end{array}$ & $\begin{array}{l}.3272 \\
.3277 \\
.3277\end{array}$ \\
\hline $\begin{array}{l}\text { KLK } \\
\text { HJK } \\
\text { MAB } \\
\text { HKH }\end{array}$ & $\begin{array}{l}\ldots \text { do } \\
\ldots \text { do } \\
\ldots\end{array}$ & $\begin{array}{l}.0008 \\
.0007 \\
.0007 \\
.0004\end{array}$ & $\begin{array}{r}.0003 \\
.0001 \\
-.0003 \\
-.0002\end{array}$ & $\begin{array}{l}.3113 \\
.3114 \\
.3114 \\
.3117\end{array}$ & $\begin{array}{l}.3289 \\
.3291 \\
.3295 \\
.3294\end{array}$ \\
\hline & & $\Delta_{0}$ & $\Delta y_{0}$ & $x_{0}$ & $y_{0}$ \\
\hline $\begin{array}{l}\text { Spectrophotomet- } \\
\text { ric. }\end{array}$ & $\left\{\begin{array}{l}\text { Rutile..... } \\
\text { Anatase... }\end{array}\right.$ & 0.0010 & -0.0008 & $\begin{array}{r}0.3153 \\
.3163\end{array}$ & $\begin{array}{r}0.3367 \\
.3359\end{array}$ \\
\hline $\begin{array}{l}\text { Colorimetric: } \\
\text { LEB } \\
\text { DBJ } \\
\text { THP }\end{array}$ & 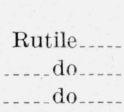 & $\begin{array}{l}.0022 \\
.0019 \\
.0019\end{array}$ & $\begin{array}{l}.0024 \\
.0019 \\
.0018\end{array}$ & $\begin{array}{l}.3141 \\
.3144 \\
.3144\end{array}$ & $\begin{array}{l}.3335 \\
.3340 \\
.3341\end{array}$ \\
\hline $\begin{array}{l}\text { KLK } \\
\text { HJK } \\
\text { MAB } \\
\text { HKH }\end{array}$ & $\ldots$ do $\ldots$ do & $\begin{array}{l}.0008 \\
.0007 \\
.0006 \\
.0003\end{array}$ & $\begin{array}{r}.0003 \\
.0001 \\
-.0004 \\
-.0003\end{array}$ & $\begin{array}{l}.3155 \\
.3156 \\
.3157 \\
.3160\end{array}$ & $\begin{array}{l}.3356 \\
.3358 \\
.3363 \\
.3362\end{array}$ \\
\hline
\end{tabular}


7 shows the result. It will be seen that both fail to meet this test by an $x$-difference of about 0.001 . It will be seen that, relative to our seven observers, the standard observer underweights the shortwave extreme of the spectrum, and the modified standard observer overweights it by about the same amount. It is evident that a modification based on an average between the standard luminosity function and the Gibson-Tyndall-Wald experimental mean would successfully meet this test within experimental uncertainty (an $x$ - or $y$ difference of 0.0003 ).

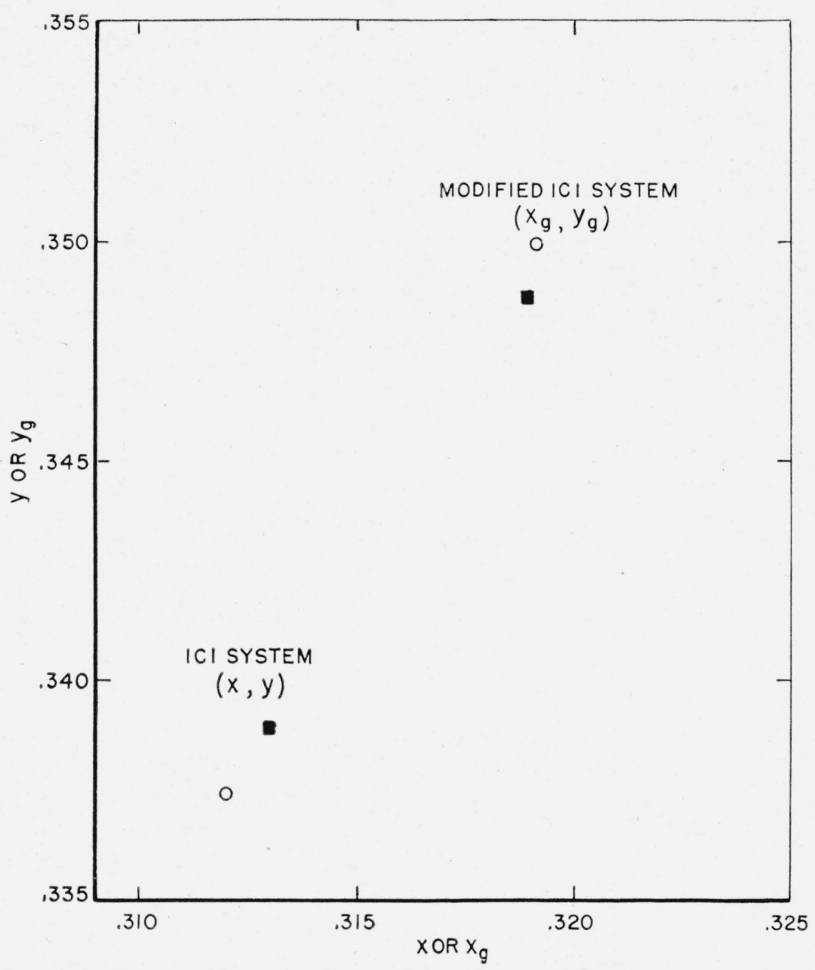

Figure 7. Comparison of the chromaticities computed from the spectral distributions of radiant flux shown in figure 6 .

Luminator: Macbeth $6,800^{\circ} \mathrm{K} ; \mathbf{\square}$, anatase $(82 \mathrm{Y}, 43 \mathrm{G}) ; \bigcirc$, rutile $(90 \mathrm{Y}$, $36 \mathrm{G})$.

The rather small size of this average discrepancy (0.001) between observed and computed chromaticity coordinates serves to emphasize the concern of the pigment industry with small color differences. This discrepancy is scarcely more than twice the least chromaticity difference that is perceptible with certainty, yet, since all observers deviate from the standard observer in the same direction, it is likely that industrial labora- tories having to interpret spectrophotometric measurements of titanium pigments will set the standard observer aside and substitute some other more or less arbitrary functions for this purpose unless steps are taken to make a suitable revision of the standard observer.

Note that the ICI $(x, y)$-diagram fails to indicate the same chromaticities for these two distributions, and that the modified $\left(x_{g}, y_{g}\right)$-diagram likewise fails, but in the opposite sense by about the same amount. This shows that an average of the ICI standard observer with the modified observer would closely account for the average result obtained by the seven observers on the chromaticity-difference colorimeter.

\section{Summary and Conclusions}

1. Jacobsen's study of the adaptability of the ICI standard observer to the indirect colorimetry of titanium dioxide pigments in linseed oil has been repeated for a single pair of specimens, one rutile, the other anatase.

2. The result of this repetition is a qualified corroboration of Jacobsen's conclusion that the ICI standard observer weights the short-wave extreme of the spectrum too lightly. Not all of our observers made settings significantly contrary to the standard observer, however. Three out of the seven obtained results in reasonable agreement with the ICI standard.

3. A modified standard observer based upon the spectral luminosity determinations of Gibson and Tyndall and of Wald has been derived. This modification is shown to account for the settings of the second group (four out of seven) much as the ICI standard accounts for those of the first group. It is presumed that the settings of Jacobsen's observers would likewise be accounted for by the modified standard observer.

4. It is suspected that if the ICI standard observer is to be maintained in its present status of general acceptability for commercial colorimetry, it may have to be modified so as to weight more heavily the short-wave extreme of the spectrum.

5. Further data relating to the luminosity of the spectrum in the wavelength region below $430 \mathrm{~m} \mu$ should be gathered to form an adequate basis for this modification. 


\section{References}

[1] A. E. Jacobsen, Non-adaptability of the ICI system to some near-whites which show absorption in the far-blue region of the spectrum, J. Opt. Soc. Am. 38, 442 (1948).

[2] Proceedings of the 8th Session, Commission Internationale de l'Éclairage, Cambridge, England, pp. 19 to 29 (1931).

[3] D. B. Judd, A Maxwell triangle yielding uniform chromaticity scales, J. Research NBS 14, 41 (1935) RP756; J. Opt. Soc. Am. 25, 31 (1935).

[4] K. S. Gibson and E. P. T. Tyndall, Visibility of radiant energy, BS Sci. Pap. 19, 131 (1923) S 475.

[5] G. Wald, Human vision and the spectrum, Science, 101, 653 (1945).
[6] Proceedings of the 6th Session, Commission Internationale de l'Éclairage, Geneva, (1924).

[7] K. S. Gibson and H. J. Keegan, Calibration and operation of the General Electric recording spectrophotometer of the National Bureau of Standards, J. Opt. Soc. Am. 28, 372 (1928).

[8] K. S. Gibson and M. M. Balcom, Transmission measurements with the Beckman quartz spectrophotometer, J. Research NBS 38, 601 (1947) RP 1798.

[9] D. B. Judd, Specification of uniform color tolerances for textiles, Textile Research 9, 253 (1939).

[10] Committee on Colorimetry, Quantitative data and methods for colorimetry, J. Opt. Soc. Am. 34, 639 (1944); table IV.

Washington, April 28, 1949. 